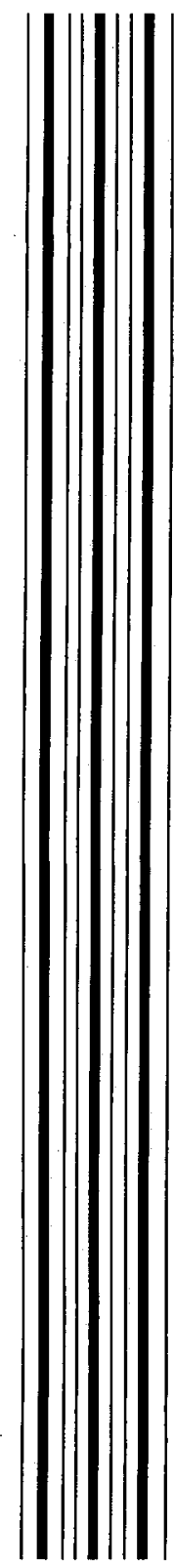

Preprint

UCRL-JC-147722

\title{
Dispersion of Extensional and Torsional Waves in Porous Cylinders with Patchy Saturation
}

\author{
J.G. Berryman, S.R. Pride
}

This article was submitted to $2^{\text {nd }}$ Conference on Poromechanics, Grenoble, France, August 26-28, 2002

U.S. Department of Energy

\section{March 20, 2002}

Lawrence

Livermore

National

Laboratory 


\section{DISCLAIMER}

This document was prepared as an account of work sponsored by an agency of the United States Government. Neither the United States Government nor the University of California nor any of their employees, makes any warranty, express or implied, or assumes any legal liability or responsibility for the accuracy, completeness, or usefulness of any information, apparatus, product, or process disclosed, or represents that its use would not infringe privately owned rights. Reference herein to any specific commercial product, process, or service by trade name, trademark, manufacturer, or otherwise, does not necessarily constitute or imply its endorsement, recommendation, or favoring by the United States Government or the University of California. The views and opinions of authors expressed herein do not necessarily state or reflect those of the United States Government or the University of California, and shall not be used for advertising or product endorsement purposes.

This is a preprint of a paper intended for publication in a journal or proceedings. Since changes may be made before publication, this preprint is made available with the understanding that it will not be cited or reproduced without the permission of the author.

This report has been reproduced directly from the best available copy.

Available electronically at http://www.doe.gov/bridge

Available for a processing fee to U.S. Department of Energy

and its contractors in paper from

U.S. Department of Energy

Office of Scientific and Technical Information

P.O. Box 62

Oak Ridge, TN 37831-0062

Telephone: (865) 576-8401

Facsimile: (865) 576-5728

E-mail: reports@adonis.osti.gov

Available for the sale to the public from

U.S. Department of Commerce

National Technical Information Service

5285 Port Royal Road

Springfield, VA 22161

Telephone: (800) 553-6847

Facsimile: (703) 605-6900

E-mail: orders@ntis.fedworld.gov

Online ordering: http://www.ntis.gov/ordering.htm

OR

Lawrence Livermore National Laboratory

Technical Information Department's Digital Library

http:/ / www.llnl.gov/tid/Library.html 


\title{
Dispersion of Extensional and Torsional Waves in Porous Cylinders with Patchy Saturation
}

\author{
James G. Berryman \\ University of California, Lawrence Livermore National Laboratory, P. O. Box 808 L-200 \\ Livermore, CA 94551-9900, USA \\ Steven R. Pride \\ Géosciences Rennes, Université de Rennes 1, Campus Beaulieu, Bât. 15 \\ 35042 Rennes Cedex, FRANCE
}

\begin{abstract}
Laboratory experiments on wave propagation through saturated and partially saturated porous media have often been conducted on porous cylinders that were initially fully saturated and then allowed to dry while continuing to acquire data on the wave behavior. Since it is known that drying typically progresses from the outside to the inside, a sensible physical model of this process is concentric cylinders having different saturation levels - the simplest example being a fully dry outer cylindrical shell together with a fully wet inner cylinder. We use this model to formulate the equations for wave dispersion in porous cylinders for patchy saturation (i.e., drainage) conditions. In addition to multiple modes of propagation obtained numerically from these dispersion relations, we find two distinct analytical expressions for torsional wave modes.
\end{abstract}

\section{INTRODUCTION}

The classic work of Pochhammer (1876) and Chree (1886) gave exact solutions for wave propagation in elastic rods. When the rod is instead a porous cylinder with fluid-filled pores, the equations of linear elasticity do not describe all possible motions of the fluid/porous-solid mixture. Biot's theory of fluid-saturated porous media provides a continuum theory, permitting the fluid and solid components to move independently and accounts approximately for the attenuation of waves due to viscous friction. Gardner (1962) used Biot's theory (Biot 1956a; Biot 1956b) to study long-wavelength extensional waves in circular cylinders. Gardner considered only the low-frequency regime where the second bulk compressional mode predicted by Biot's theory is diffusive in character. Gardner also limited consideration to the case of open-pore surface boundary conditions.

The present work is based in part on another paper by Berryman (1983), in which both openpore and closed-pore surface boundary conditions for the fluid-saturated porous cylinder were studied. Here we consider only the open-pore surface, but we allow non-uniform or patchy saturation (Berryman et al. 1988; Knight and Nolen-Hoeksema 1990; Knight et al. 1998; Johnson 2001) inside the cylinder. In particular, it is quite common to study partial saturation in the laboratory under drainage or drying conditions wherein an initially fully saturated porous cylinder is allowed to dry while continuing to acquire data on the cylinder's modes of oscillation. We want to model this behavior explicitly. The simplest such model is concentric cylinders with a fully dry outer cylindrical 
shell enclosing a fully liquid-saturated inner cylinder.

We present the equations of poroelasticity, and then show the forms of the equations needed for cylindrical geometry. Appropriate boundary conditions for our problem are discussed. Equations are subsequently formulated to determine the extensional and torsional modes of concentric poroelastic cylinders under conditions of partial saturation.

\section{EQUATIONS OF POROELASTICITY}

For long-wavelength disturbances ( $\lambda>>h$ where $h$ is a typical pore size) propagating through such a porous medium, we define average values of the (local) displacements in the solid and also in the saturating fluid. The average displacement vector for the solid frame is $\mathbf{u}$ while that for the pore fluid is $\mathbf{u}_{f}$. The average displacement of the fluid relative to the frame is $\mathbf{w}=\phi\left(\mathbf{u}-\mathbf{u}_{f}\right)$. For small strains, the frame dilatation is

$$
e=e_{x}+e_{y}+e_{z}=\nabla \cdot \mathbf{u}
$$

where $e_{x}, e_{y}, e_{z}$ are the Cartesian strain components. Similarly, the average fluid dilatation is

$$
e_{f}=\nabla \cdot \mathbf{u}_{f}
$$

( $e_{f}$ also includes flow terms as well as dilatation) and the increment of fluid content is defined by

$$
\zeta=-\nabla \cdot \mathbf{w}=\phi\left(e-e_{f}\right)
$$

With these definitions, Biot (1962) shows that the strain-energy functional for an isotropic, linear medium is a quadratic function of the strain invariants (Love 1944) $I_{1}=e, I_{2}$, and of $\zeta$ having the form

$$
2 E=H e^{2}-2 C e \zeta+M \zeta^{2}-4 \mu I_{2}
$$

where

$$
I_{2}=e_{x} e_{y}+e_{y} e_{z}+e_{z} e_{x}-\frac{1}{4}\left(\gamma_{x}^{2}+\gamma_{y}^{2}+\gamma_{z}^{2}\right)
$$

and $\gamma_{x}, \gamma_{y}, \gamma_{z}$ are the shear strain components. The stress-strain relations which follow from (4) are

$$
\tau_{x x}=\frac{\partial E}{\partial e_{x}}=H e-2 \mu\left(e_{y}+e_{z}\right)-C \zeta,
$$

and similarly for $\tau_{y y}, \tau_{z z}$, while

$$
\tau_{z x}=\frac{\partial E}{\partial \gamma_{y}}=\mu \gamma_{y}
$$

and similarly for $\tau_{y z}, \tau_{x y}$, and finally

$$
p_{f}=\frac{\partial E}{\partial \zeta}=M \zeta-C e .
$$

The $\tau_{i j}$ are average Cartesian stresses in the saturated porous material and $p_{f}$ is the isotropic pressure in the pore fluid. 
With time dependence of the form $\exp (-i \omega t)$, the coupled wave equations that follow from (6)-(8) in the presence of dissipation are

$$
\begin{gathered}
\omega^{2}\left(\rho \mathbf{u}+\rho_{f} \mathbf{w}\right)=C \nabla \zeta-(H-\mu) \nabla e-\mu \nabla^{2} \mathbf{u} \\
\omega^{2}\left(\rho_{f} \mathbf{u}+q \mathbf{w}\right)=M \nabla \zeta-C \nabla e
\end{gathered}
$$

where $\rho=\phi \rho_{f}+(1-\phi) \rho_{m}$ and $q=\rho_{f}[\alpha / \beta+i F(\xi) \eta / \kappa \omega]$. The kinematic viscosity of the liquid is $\eta$; the permeability of the porous frame is $\kappa$; the dynamic viscosity factor is given, for our choice of sign for the frequency dependence, by $F(\xi)=\frac{1}{4}\{\xi T(\xi) /[1+2 T(\xi) / i \xi]\}$, where $T(\xi)=$

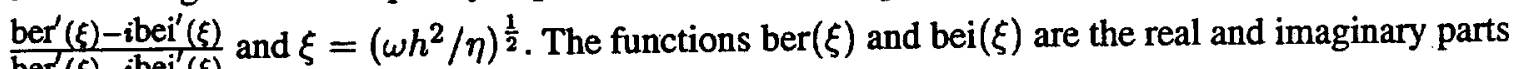
of the Kelvin function. The dynamic parameter $h$ is a characteristic length generally associated with and comparable in magnitude to the steady-flow hydraulic radius. The tortuosity $\alpha \geq 1$ is a pure number related to the frame inertia which has been measured by Johnson et al. (1982) and has also been estimated theoretically by Berryman (1980).

The coefficients $H, C$, and $M$ are given by (Gassmann 1951; Biot and Willis 1957; Geertsma and Smit 1961; Stoll 1974)

$$
\begin{gathered}
H=K+\frac{4}{3} \mu+\left(K_{m}-K\right)^{2} /(D-K) \\
C=K_{m}\left(K_{m}-K\right) /(D-K),
\end{gathered}
$$

and

$$
M=K_{m}^{2} /(D-K)
$$

where

$$
D=K_{m}\left[1+\phi\left(K_{m} / K_{f}-1\right)\right]
$$

Equations (10)-(13) are correct as long as the porous material may be considered homogeneous on the microscopic scale as well as the macroscopic scale.

To decouple the wave equations (9) into Helmholtz equations for the three modes of propagation, we note that the displacements $\mathbf{u}$ and $\mathbf{w}$ can be decomposed as

$$
\mathbf{u}=\nabla \Upsilon+\nabla \times \vec{\beta}, \quad \mathbf{w}=\nabla \psi+\nabla \times \vec{\chi},
$$

where $\Upsilon, \psi$ are scalar potentials and $\vec{\beta}, \vec{\chi}$ are vector potentials. Substituting (14) into (9), we find $(9)$ is satisfied if two pairs of equations are satisfied:

$$
\left(\nabla^{2}+k_{s}^{2}\right) \vec{\beta}=0, \quad \vec{\chi}=-\rho_{f} \vec{\beta} / q
$$

and

$$
\left(\nabla^{2}+k_{ \pm}^{2}\right) A_{ \pm}=0
$$

The wave vectors in (15) and (16) are defined by

$$
k_{s}^{2}=\omega^{2}\left(\rho-\rho_{f}^{2} / q\right) / \mu
$$


and

$$
\begin{gathered}
k_{ \pm}^{2}=\frac{1}{2}\left\{b+f \mp\left[(b-f)^{2}+4 c d\right]^{\frac{1}{2}}\right\} \\
b=\omega^{2}\left(\rho M-\rho_{f} C\right) / \Delta, c=\omega^{2}\left(\rho_{f} M-q C\right) / \Delta \\
d=\omega^{2}\left(\rho_{f} H-\rho C\right) / \Delta, f=\omega^{2}\left(q H-\rho_{f} C\right) / \Delta,
\end{gathered}
$$

with $\Delta=M H-C^{2}$. The linear combination of scalar potentials has been chosen to be $A_{ \pm}=$ $\Gamma_{ \pm} \Upsilon+\psi$, where

$$
\Gamma_{ \pm}=d /\left(k_{ \pm}^{2}-b\right)=\left(k_{ \pm}^{2}-f\right) / c
$$

With the identification (20), the decoupling is complete.

Equations (15) and (16) are valid for any choice of coordinate system. They may be applied to boundary value problems with arbitrary symmetry. Biot's theory of poroelasticity will therefore be applied to cylinders in the next section.

\section{EQUATIONS FOR A POROUS CYLINDER}

To work most easily in cylindrical geometry, we rewrite the stress-strain relations (6)-(8) in cylindrical coordinates. If $z$ is the coordinate along the cylinder axis while rand $\theta$ are the radial and azimuthal coordinates, it is not difficult to show that

$$
\begin{gathered}
\tau_{r r}=\frac{\partial E}{\partial e_{r}}=H e-2 \mu\left(e_{\theta}+e_{z}\right)-C \zeta \\
\tau_{r \theta}=\mu\left(\frac{\partial u_{\theta}}{\partial r}-\frac{u_{\theta}}{r}+\frac{1}{r} \frac{\partial u_{r}}{\partial \theta}\right) \\
\tau_{r z}=\mu\left(\frac{\partial u_{r}}{\partial z}+\frac{\partial u_{z}}{\partial r}\right)
\end{gathered}
$$

and (8) remains unchanged. The stress components $\tau_{z z}, \tau_{\theta \theta}$, and $\tau_{\theta z}$ are not of direct interest in the present application. The dilatations are given by

$$
e=e_{r}+e_{\theta}+e_{z}
$$

where

$$
e_{r}=\frac{\partial u_{r}}{\partial r}, \quad e_{\theta}=\frac{u_{r}}{r}+\frac{1}{r} \frac{\partial u_{\theta}}{\partial \theta}, \quad e_{z}=\frac{\partial u_{z}}{\partial z}
$$

We redefine potential $\vec{\beta}$ in terms of two scalar potentials according to

$$
\vec{\beta}=\hat{z} \beta_{1}+\nabla \times\left(\hat{z} \beta_{2}\right),
$$

where both $\beta_{i}$ satisfy

$$
\left(\nabla^{2}+k_{s}^{2}\right) \beta_{i}=0 \quad \text { for } \quad i=1,2
$$


For the problem of interest here, we will have two distinct regions: The first region is a cylinder centered at the origin, within which solutions of (16) and (27) must be finite at the origin. Results take the form

$$
\begin{gathered}
A_{ \pm}=\alpha_{ \pm} J_{0}\left(j_{ \pm}\right) \exp i\left(k_{z} z-\omega t\right), \\
\beta_{1}=\gamma_{s} J_{0}\left(j_{s}\right) \exp i\left(k_{z} z-\omega t\right), \\
\beta_{2}=\left(\alpha_{s} / i k_{z}\right) J_{0}\left(j_{s}\right) \exp i\left(k_{z} z-\omega t\right),
\end{gathered}
$$

where

$$
j_{ \pm}=k_{ \pm r} r, \quad j_{s}=k_{s r} r
$$

and

$$
k_{ \pm r}^{2}=k_{ \pm}^{2}-k_{z}^{2}, \quad k_{s r}^{2}=k_{s}^{2}-k_{z}^{2}
$$

$J_{0}$ is the zero-order Bessel function of the first kind. The coefficients $\alpha_{ \pm}, \alpha_{s}, \gamma_{s}$, are constants to be determined from the boundary conditions.

The second region is a cylindrical shell around the first region. In this region, the factors $k_{ \pm}$ and $k_{s}$ take different values from the those in the central region, indicated by $k_{ \pm}^{*}$ and $k_{s}^{*}$ (where * means air-filled, and does not ever mean complex conjugate in this paper). Furthermore, two linearly independent solutions of the equations are allowed, i.e., both $J_{0}$ and $Y_{0}$ (the Bessel function of the second kind, sometimes known as the Neumann function). In the outer shell, we have four coefficients for $J_{0}$ and four for $Y_{0}$, all of which must also be determined by the boundary conditions.

Noting that

$$
\begin{aligned}
& \Upsilon=\left(A_{+}-A_{-}\right) /\left(\Gamma_{+}-\Gamma_{-}\right) \\
& \psi=\left(A_{+} \Gamma_{-}-A_{-} \Gamma_{+}\right) /\left(\Gamma_{-}-\Gamma_{+}\right)
\end{aligned}
$$

from the definitions of $A_{ \pm}$, and substituting (28)-(30) and (33) into (14), and the result into (8) and (21)-(23), we finally obtain

$$
\tau_{r \theta}=m_{11} \gamma_{s} \equiv \mu k_{s r}^{2}\left[J_{0}\left(j_{s}\right)-2 J_{1}\left(j_{s}\right) / j_{s}\right] \gamma_{s}
$$

and

$$
\begin{aligned}
& \tau_{r r}=a_{11} \alpha_{+}+a_{12} \alpha_{-}+a_{13} \alpha_{s} \\
& -p_{f}=a_{21} \alpha_{+}+a_{22} \alpha_{-}+a_{23} \alpha_{s} \\
& \tau_{r z}=a_{31} \alpha_{+}+a_{32} \alpha_{-}+a_{33} \alpha_{s}
\end{aligned}
$$

where

$$
\begin{gathered}
\left(\Gamma_{+}-\Gamma_{-}\right) a_{11}=\left[\left(C \Gamma_{-}-H\right) k_{+}^{2}+2 \mu k_{z}^{2}\right] J_{0}\left(j_{+}\right) \\
+2 \mu k_{+r} J_{1}\left(j_{+}\right) / r
\end{gathered}
$$




$$
\begin{gathered}
\left(\Gamma_{+}-\Gamma_{-}\right) a_{12}=-2 \mu k_{-r}^{2} J_{1}\left(j_{-}\right) / j_{-} \\
+\left[\left(H-C \Gamma_{+}\right) k_{-}^{2}-2 \mu k_{z}^{2}\right] J_{0}\left(j_{-}\right), \\
a_{13}=-2 \mu k_{s r}^{2}\left[J_{0}\left(j_{s}\right)-J_{1}\left(j_{s}\right) / j_{s}\right], \\
\left(\Gamma_{+}-\Gamma_{-}\right) a_{21}=\left(M \Gamma_{-}-C\right) k_{+}^{2} J_{0}\left(j_{+}\right), \\
\left(\Gamma_{+}-\Gamma_{-}\right) a_{22}=\left(C-M \Gamma_{+}\right) k_{-}^{2} J_{0}\left(j_{-}\right), \\
\left(\Gamma_{+}-\Gamma_{-}\right) a_{31}=-2 i \mu k_{z} k_{+r} J_{1}\left(j_{+}\right), \\
\left(\Gamma_{+}-\Gamma_{-}\right) a_{32}=2 i \mu k_{z} k_{-r} J_{1}\left(j_{-}\right), \\
i k_{z} a_{33}=-\mu\left(k_{s}^{2}-2 k_{z}^{2}\right) k_{s r} J_{1}\left(j_{s}\right),
\end{gathered}
$$

and $a_{23}=0$. There is an implicit factor of $\exp i\left(k_{z} z-\omega t\right)$ on the right-hand side of (34)-(37).

Berryman (1983) has shown that $a_{11}, a_{13}, a_{31}$, and $a_{33}$ reduce in the limit $\phi \rightarrow 0$ to the corresponding results for isotropic elastic cylinders by Pochhammer (1876), Chrèe (1886), Chree (1889), Love (1944), and Bancroft (1941), as they should.

\section{BOUNDARY CONDITIONS}

While appropriate boundary conditions for use with Biot's equations are not obvious, Deresiewicz and Skalak (1963), Berryman and Thigpen (1985), and Pride and Haartsen (1996) have studied boundary conditions for saturated and partially saturated porous media and we make use of these results here.

Deresiewicz and Skalak (1963) show that, if $n_{j}$ is the $j$ th component of the unit normal to the interface, the expression

$$
\left(\tau_{i j} \dot{u}_{i}-p_{f} \delta_{i j} \dot{w}_{i}\right) n_{j}
$$

must be continuous across the interface. The surface integral of (46) is the rate at which work is done on the material by forces acting on its surface. If the fluids inside and outside the porous material are the same and if the surface pores are open, the boundary conditions (Deresiewicz and Skalak 1963)

$$
\tau_{n n}=-p_{f}=-\bar{p}_{f}, \quad \tau_{n s}=0
$$

and

$$
\dot{u}_{n}+\dot{w}_{n}=\dot{\bar{U}}_{n}
$$

guarantee continuity of (46). The pressure and displacement of the exterior fluid are $\bar{p}_{f}$ and $\bar{U}$. In (47)-(48), the subscript $n$ indicates a normal component, while $s$ indicates a shear component tangential to the surface. We take these conditions (47) and (48) to apply at the exterior boundary of the outer (air-filled) cylindrical shell. 
If the inner liquid-saturated porous cylinder is surrounded by a poroelastic material with a different fluid saturating the pores (air in this case), the boundary conditions at the interface between liquid-filled and air-filled regions are (Deresiewicz and Skalak 1963)

$$
\begin{array}{cc}
\tau_{n n}=\tau_{n n}^{*}, & \tau_{n s}=\tau_{n s}^{*}, \\
\dot{u}_{n}=\dot{u}_{n}^{*}, & \dot{u}_{s}=\dot{u}_{s}^{*}
\end{array}
$$

and

$$
\dot{w}_{n}=\dot{w}_{n}^{*}, \quad p_{f}=p_{f}^{*}
$$

The solid stress and displacement in the adjacent poroelastic material are $\tau^{*}$ and $u^{*}$, and the corresponding increment of fluid content and pressure are $w_{n}^{*}$ and $p_{f}^{*}$. All these conditions are required to assure continuity of (46) at the inner boundary.

To apply the boundary conditions (51), we need in addition to (36) the result

$$
w_{r}=a_{41} \alpha_{+}+a_{42} \alpha_{-}+a_{43} \alpha_{s}
$$

where

$$
\begin{gathered}
\left(\Gamma_{+}-\Gamma_{-}\right) a_{41}=k_{+r} J_{1}\left(j_{+}\right) \Gamma_{-} \\
\left(\Gamma_{+}-\Gamma_{-}\right) a_{42}=-k_{-r} J_{1}\left(j_{-}\right) \Gamma_{+} \\
a_{43}=k_{s r} J_{1}\left(j_{s}\right) \rho_{f} / q .
\end{gathered}
$$

The remaining stress conditions (49) are determined by (35) and (37).

To apply the boundary conditions (50), we need the explicit expressions for the displacement which follow from (14). The results are of the form

$$
u_{r}=a_{51} \alpha_{+}+a_{52} \alpha_{-}+a_{53} \alpha_{s}
$$

where

$$
\begin{gathered}
\left(\Gamma_{+}-\Gamma_{-}\right) a_{51}=-k_{+r} J_{1}\left(j_{+}\right) \\
\left(\Gamma_{+}-\Gamma_{-}\right) a_{52}=k_{-r} J_{1}\left(j_{-}\right) \\
a_{53}=k_{s r} J_{1}\left(j_{s}\right)
\end{gathered}
$$

and

$$
u_{z}=a_{61} \alpha_{+}+a_{62} \alpha_{-}+a_{63} \alpha_{s}
$$

where $a_{61}=a_{62}=0$, and

$$
a_{63}=k_{s r}^{2} J_{0}\left(j_{s}\right) / i k_{z}
$$




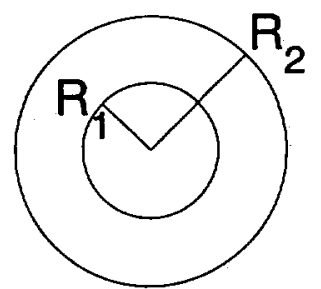

FIG. 1. Cross-section of a circular cylinder, where $R_{1}=S^{\frac{1}{2}} R_{2}$ is determined by the liquid saturation level $S$.

Both (56) and (60) are needed for extensional waves, while the remaining component,

$$
u_{\theta}=m_{21} \gamma_{s} \equiv k_{s r} J_{1}\left(j_{s}\right) \gamma_{s}
$$

is needed only for torsional waves. As before, there is an implicit factor of $\exp i\left(k_{z} z-\omega t\right)$ on the right-hand side of (53)-(55), (57)-(59), and (61).

It follows from (34)-(37), (52), and (62) that $\gamma_{s}$ (for the inner cylinder) and the corresponding coefficients for the cylindrical shell are all completely independent of the other mode coefficients and, therefore, relevant to the study of torsional waves, but not for extensional waves. Pertinent equations for the torsional wave dispersion relation are continuity of the angular displacement $u_{\theta}$ and stress $\tau_{r} \theta$ at the internal interface, and vanishing of the stress $\tau_{r} \theta$ at the external surface.

The final set of equations for the extensional wave dispersion relation involves nine equations in nine unknowns: The nine unknowns are $\alpha_{+}, \alpha_{-}, \alpha_{s}$ (coefficients of $J_{0}$ in the central cylinder), plus three $\alpha^{*}$ 's (coefficients of $J_{0}$ ) and three $\eta^{*}$ 's (coefficients of $Y_{0}$ ) for region of the cylindrical shell. The nine equations are continuity of radial and one tangential stress as well as radial and one tangential displacement at the interfacial boundary (totaling four conditions), continuity of fluid pressure and normal fluid increments across the same boundary (two conditions), and finally the vanishing of the external fluid pressure, radial and one tangential stress at the free surface (three conditions). The extensional wave dispersion relation is then determined as in Berryman (1983) by those conditions on the wavenumber $k_{z}$ that result in vanishing of the determinant of the coefficients of this $9 \times 9$ complex matrix.

\section{TORSIONAL WAVE EXAMPLE}

Of the 81 elements in the matrix determining the extensional dispersion relation, there are 69 that will in general be nonzero. Length constraints on the present manuscript will therefore preclude further studies of this mode here. On the other hand, the torsional mode (which is trivial for a simple cylinder) now is determined by a $3 \times 3$ system, of which 8 elements are in general nonzero. This system is therefore similar in size and difficulty to the cases studied earlier by Berryman (1983) for extensional waves in a simple poroelastic cylinder.

We assume that the cylinder has liquid saturation level $S=\left(R_{1} / R_{2}\right)^{2}$, where $R_{2}$ is the radius of the cylinder and $r=R_{1}$ is the location of the liquid-gas interface (see Fig. 1). The dispersion relation for torsional waves is then given by

$$
\left|\begin{array}{ccc}
m_{11}^{*}\left(R_{2}\right) & n_{11}^{*}\left(R_{2}\right) & 0 \\
-m_{11}^{*}\left(R_{1}\right) & -n_{11}^{*}\left(R_{1}\right) & m_{11}\left(R_{1}\right) \\
-m_{21}^{*}\left(R_{1}\right) & -n_{21}^{*}\left(R_{1}\right) & m_{21}\left(R_{1}\right)
\end{array}\right|=0
$$


where $m_{11}$ and $m_{21}$ are given by (34) and (62). The coefficients $m_{11}^{*}$ and $m_{12}^{*}$ have the same functional forms as $m_{11}$ and $m_{21}$, but the constants are those for the shell, rather than the inner cylinder. Similarly, $n_{11}^{*}$ and $n_{12}^{*}$ are just the same as $m_{11}^{*}$ and $m_{12}^{*}$ except that $J_{0}$ and $J_{1}$ are replaced everywhere by $Y_{0}$ and $Y_{1}$, respectively.

Now we notice immediately that there could be two elementary solutions of (63), one when $m_{11}^{*}\left(R_{2}\right)=n_{11}^{*}\left(R_{2}\right)=0$ (exterior condition) and another when $m_{11}\left(R_{1}\right)=m_{21}\left(R_{1}\right)=0$ (interior condition). First, the interior condition is satisfied, for example, when $k_{s r}=0$ or, equivalently, when $k_{z}^{2}=k_{s}^{2}$. This corresponds to a torsional mode of propagation having wave speed and attenuation determined exactly by the bulk shear wave in the interior region, but the interior region is not moving since $k_{s r}=0$ also implies that $u_{\theta}=0$ from (62). Thus, the interior condition results in the drained shell twisting around a stationary inner liquid-saturated cylinder. Second, the exterior condition is similarly satisfied when $k_{s r}^{*}=0$ or, equivalently, when $k_{z}^{2}=\left(k_{s}^{*}\right)^{2}$. This condition looks at first glance as if it might be spurious because $k_{s r}^{*}=0$ suggests that $u_{\theta}$ at the exterior boundary might vanish identically, and then this would correspond to a trivial solution of the equations. However, looking closer, this is not the case, because at the external boundary

$$
u_{\theta}=k_{s r}^{*}\left[J_{1}\left(j_{s}^{*}\right) \gamma_{s}^{*}+Y_{1}\left(j_{s}^{*}\right) \epsilon_{s}^{*}\right]
$$

so as $k_{s r}^{*} \rightarrow 0$, the first term on the right hand side of (64) does vanish, both because $k_{s r}^{*} \rightarrow 0$ and also because $J_{1}\left(j_{s}^{*}\right) \rightarrow 0$. But the second term does not vanish in this limit because $\left|Y_{1}\left(j_{s}^{*}\right)\right| \rightarrow$ $2 / \pi k_{s r}^{*} R_{2} \rightarrow \infty$ as $k_{s r}^{*} \rightarrow 0$, and the product gives the finite result: $2 / \pi R_{2}$. So this condition is not spurious, and corresponds to a torsional wave propagating with the speed and attenuation of the bulk shear wave speed in the drained shell material.

The important conclusion is that arbitrary linear combinations of these two elementary solutions can be excited in laboratory torsional experiments and these results must therefore be used to analyze data from drainage experiments.

\section{SUMMARY}

Biot slow-wave effects in layered materials have been studied previously by Pride et al. (2002) and many others found in their references. The present work is motivated by the desire to understand how fluids interacting with common poroelastic systems may create viscous attenuation in partially saturated (and especially in patchy saturated) cylinders. These effects can then be observed in the attenuation of extensional and torsional waves. There are large quantities of such data already available, and one thrust of our future work will be to analyze these data in light of the methods developed here.

\section{ACKNOWLEDGMENTS}

Work of JGB was performed under the auspices of the U.S. Department of Energy under contract No. W-7405-ENG-48 and supported specifically by the Geosciences Research Program of the DOE Office of Basic Energy Sciences, Division of Chemical Sciences, Geosciences and Biosciences.

\section{REFERENCES}

Bancroft, D. (1941). "The velocity of longitudinal waves in cylindrical bars.” Phys. Rev., 59, 588593.

Berryman, J. G. (1980). “Confirmation of Biot's theory.” Appl. Phys. Lett., 37, 382-384.

Berryman, J. G. (1983). "Dispersion of extensional waves in fluid-saturated porous cylinders at ultrasonic frequencies." J. Acoust. Soc. Am., 74, 1805-1812. 